\title{
Diabetic Retinopathy Screening Using Computer Vision
}

\author{
Christopher E. Hann*, J. Geoffrey Chase*, James A. Revie*, Darren Hewett*, Geoffrey M. Shaw** \\ *University of Canterbury, Dept of Mechanical Engineering, Christchurch, New Zealand \\ (Tel: +64-3-364-7001; e-mail: chris.hann@ Canterbury.ac.nz). \\ **Dept of Intensive Care, Christchurch Hospital, Christchurch, New Zealand \\ (e-mail:geoff.shaw@cdhb.govt.nz)
}

\begin{abstract}
Diabetic Retinopathy (DR) is one of the main causes of blindness and visual impairment in developed countries, stemming solely from diabetes mellitus. Current screening methods using fundus images rely on the experience of the operator as they are manually examined. Automated methods based on neural networks and other approaches have not provided sensitivity or specificity above $85 \%$. This work presents a computer vision based method that directly identifies hard exudates and dot haemorrhages $(\mathrm{DH})$ from 100 digital fundus images from a graded database of images using standard computer vision techniques, and clinical observation and knowledge. Sensitivity and specificity in diagnosis are 95-100\% in both cases. Positive and negative prediction values (PPV, NPV) were 95-100\% for both cases. The overall method is general, computationally efficient and suitable for further clinical trials to test both accuracy and the ability to the track DR status over time.
\end{abstract}

Keywords: Computer Vision, Diabetes, Retinopathy, Diagnosis, Automation.

\section{INTRODUCTION}

Diabetic Retinopathy (DR) is one of the main causes of blindness and visual impairment in developed countries (Icks et al, 1997; Fong et al, 2004). The prevalence of retinopathy and vision threatening retinopathy in the US are $40.3 \%$ and $8.2 \%$ respectively for diabetic adults 40 years or older (TEDPRG, 2004). The number of people with diabetes is expected to double in the next 15-30 years due to obesity, aging populations and inactive lifestyles, with over $80 \%$ of diabetic individuals affected by DR (Wild et al, 2004). However, early detection combined with appropriate treatment and management can prevent the loss of vision in up to $95 \%$ of cases (Fong et al, 2003, 2004; Chia et al, 2004; ETDRSRG, 1991).

The high prevalence of diabetes therefore makes mass screening an expensive and time consuming process. An automated system could greatly reduce the workload by filtering out $50 \%$ of the screening population (Hipwell et al, 2000). However, a recent 11,000 patient study concluded that automated detection of diabetic retinopathy using published algorithms cannot yet be recommended for clinical practice (Abramoff et al, 2008), indicating a significant need for improvement in this arena.

DR results from leakage of small vessels in the retina due to hyperglycaemia. In the early stages, known as nonproliferated retinopathy, there may be haemorrhages due to bleeding of the capillaries, or exudates resulting from protein deposits in the retina. There is usually no vision loss unless there is a build up of fluid in the centre of the eye. As the disease progresses, new abnormal vessels grow in the retina, known as neovascularisation. These vessels frequently leak into the vitreous, which is called proliferated retinopathy and may cause severe visual problems. The goal of screening is to detect non-proliferated DR to implement management that decreases the chances of (long-term) vision impairment.

The use of seven-field stereo fundus photography read by a trained reader is the gold standard diagnostic. DR grading using the fundus images is significantly more sensitive than standard opthalmoscopy, which can miss approximately $50 \%$ of subjects with only microaneurysms, resulting in under reporting of DR by $\sim 10 \%$ (Kinyoun et al, 1992; Moss et al, 1985). Recent research has combined fundus photography and computer algorithms to grade DR (Abramoff et al, 2008). The algorithms search for bad lesions in the fundus images which define the severity of DR. The lesions are categorised into microaneurysms, haemorrhages and exudates based on their location, morphology and colour. Several recent reports on detecting one of these lesion types report sensitivities of $81.3-90.1 \%$ with false positive rates of $\sim 18 \%$ (Sanchez et al, 2007; Walter et al, 2007; Sinthnayothin et al, 2002). The high false positive rates are the main issue with current computer vision based screening methods. The most common classifiers used with computer vision are neural networks or statistical classifiers (Frame et al, 1998; Gardner et al, 1996).

This paper takes an alternative more analytical approach by focusing on direct identification using accurate geometric models at the preprocessing stage. Two separate algorithms are developed to detect exudates and dot haemorrhages. Information from the colour, morphology and intensity gradients of the fundus photographs provide a means to detect the number of exudates and dot haemorrhages and thus determine the presence of DR. This paper therefore focuses on the problem of accurately detecting DR rather than the grading of images. However, the methods presented are general enough to enable monitoring of the patients DR status over time given suitable images. Thus, computer vision may offer more than the current clinical, manual approach. 


\section{METHODS}

Two separate algorithms are written. One each for automatically detecting exudates and dot haemorrhages from fundus images.

\subsection{Identifying Hard Exudates:}

Exudates are common abnormalities in the retina, and are bright lipid leaked from a blood vessel. The leaked fluid tends to stay close to the puncture yielding a generally welldefined edge suitable for computer analysis (Ege et al, 2000). Figure 1 gives an example of exudates on a fundus image, which show up as small, light yellow regions. However the optic disc, which can be seen in Figure 1, is also a light yellow region.

Therefore, before searching for exudates, an algorithm is developed for automatic detection of the optic disc. In the image, the yellow colour corresponds to a high intensity on the green channel and typically the optic disc contains the majority of the highest green intensities on a given image. Thus, an initial approximation to the optic disc is obtained by sorting the green intensities from the lowest to the highest, and choosing all pixels in the top $0.5 \%$ of intensities. This method may also capture some other bright yellow regions like exudates, but the majority will lie on the optic disc. Figure 1 shows the optic disk identified as the largest connected region (LCR) of such dots on this color channel, as well as the bounding circle created to eliminate it from further consideration in the algorithm.

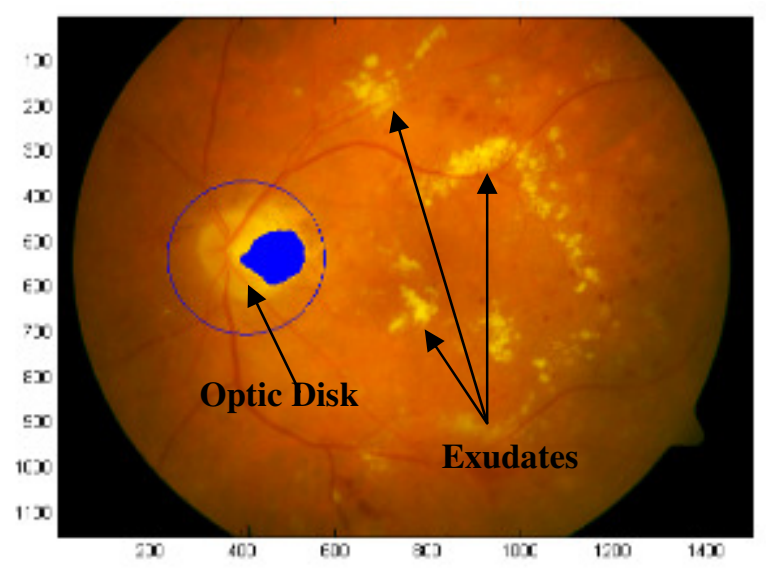

Figure 1: Optic disk, LCR and hard exudates identified on a fundus image via yellow color. The LCR is identified in blue and doesn't cover the entire optic disk, but the identified portion that is not searched as a result is circled.

In identifying the other exudates in Figure 1, or any image, each region found must be bound by a contour to identify if it is 1 large exudate or several smaller exudates. Such contours would thus help identify, over time, the growth of current exudates and the number of any new exudates.

The neighbourhood of an exudate is defined as a rectangle with a boundary 40 pixels from the unique rectangle precisely surrounding the exudate. Within the exudate neighbourhood, contours of $\mathrm{red} / \mathrm{green}(\mathrm{R} / \mathrm{G})$ intensity are computed around the mean $R / G$ of the exudate. Contours of sufficient length and "nearby" are selected as potential exudates. Nearby is defined on the distance from the centre of the unique circle, with radius $\mathrm{r}_{\text {EXUDATE }}$ precisely surrounding the exudate. Specifically, if any part of the contour is within a radius of $1.5^{*} \mathrm{r}_{\text {EXUDATE }}$ the contour is selected as a candidate for the boundary of exudate. Once all the "nearby" contours are selected, the contour with the largest mean absolute image gradient is chosen. In addition, the contour must have a length of greater than 20 pixels to be large enough for consideration.

The overall algorithm is based on specific colour channels and standard image processing techniques in a new configuration. The algorithm is defined:

\section{Define Optic Disk:}

- Obtain top $0.5 \%$ of pixel intensities in green channel

- Place a rectangle around the largest connected region

- Define a circle with the centre at the left edge mid-point and radius of the horizontal width respectively

- Increase radius 50\% to guarantee disk is bounded

\section{Find Exudates Using Median Filter:}

- Compute 50 pixel median filter along all vertical and horizontal lines. Median filter takes the moving median intensity value over 50 pixels (centered).

- Subtract the minimum of the median in each direction. From each pixel intensity to equalize the contrast over the image.

- For each vertical and horizontal line, select pixels with filtered intensities $>10$ and take the union. If the filtered intensities $>30$ and the green intensity $>100$, label the pixel $\mathrm{I}_{\mathrm{CE}}$, where $\mathrm{CE}=$ "confirmed exudate".

- Form connected regions, remove regions with number of pixels $<5$. Remove regions containing pixels with red + green + blue channel $<60$, remove regions containing pixels in optic disc.

\section{Add Pixels and Confirm Bright Exudates}

- $\quad$ For each exudate compute the lower 5 th percentile $\mathrm{I}_{\text {low }}$ and upper 95th percentile $I_{\text {high }}$ of red/green $(\mathrm{R} / \mathrm{G})$ intensities. Within a \pm 100 pixel neighbourhood of the exudate, select pixels where: $(\mathrm{R} / \mathrm{G}) \varepsilon\left[\mathrm{I}_{\text {low }}, \mathrm{I}_{\text {high }}\right]$. Add the selected pixels to the potential exudate if they are connected to the exudate.

- Save all connected regions that have pixels labelled $\mathrm{I}_{\mathrm{CE}}$. Remove these regions from the image analysis as they are confirmed as exudates.

\section{Remove False Exudates:}

- In a 40-pixel neighbourhood of each remaining potential exudate, compute the $\mathrm{R} / \mathrm{G}$ contours in steps of $0.05 \pm 0.5$ around the mean $\mathrm{R} / \mathrm{G}$ for the exudate.

- Let $\mathrm{r}_{\text {EXUDATE }}$ denote the radius of the unique circle, $\mathrm{C}$, that precisely surrounds the exudate. Select a contour if:

a) Any part of contour is within $1.5^{*} \mathrm{r}_{\text {EXUDATE }}$ from 
the centre of the circle

b) The contour has a length $>20$ pixels

- For each selected contour, C, calculate the mean absolute gradient of the surrounding points and choose the contour corresponding to the largest value

- Compute the distance $\mathrm{D}_{\mathrm{c}}$ between the current contour and nearest exudate. If $D_{c}>4$ pixels, reject the exudate

\section{Output Number and Size of All Exudates:}

\subsection{Identifying Dot Haemorrhages:}

Haemorrhages are a secondary sign of diabetic retinopathy resulting from ruptured micro aneurysms, capillaries and venules. Classification of dot haemorrhages (DHs) depends on their location within the retinal layers. DHs are located within the outer plexiform and inner nuclear layer. The round shape and distinct borders of DHs are due to intra-retinal compression confining the $\mathrm{DH}$ to a specific location.

DHs have a similar red colour to the vessels and are close to circular with well defined boundaries. This circularity is used to segment the DHs from other features in the fundus image. The colour similarity between DHs and vessels, makes it necessary to accurately detect the vessels from the image before checking for DHs.

The ratio of the red to green $(\mathrm{R} / \mathrm{G})$ intensities gives the best definition of the red features, and is also more resistant to lighting changes between images. The $\mathrm{R} / \mathrm{G}$ ratio brightens the vessels, DHs and the fovea, which are predominantly red, and dulls the primarily yellow optic disk and exudates. An example image of the $\mathrm{R} / \mathrm{G}$ ratio is shown in Figure 2, where the significant brightening of the $\mathrm{DH}$ and vessels simplifies the choice of thresholds to select the desired regions.

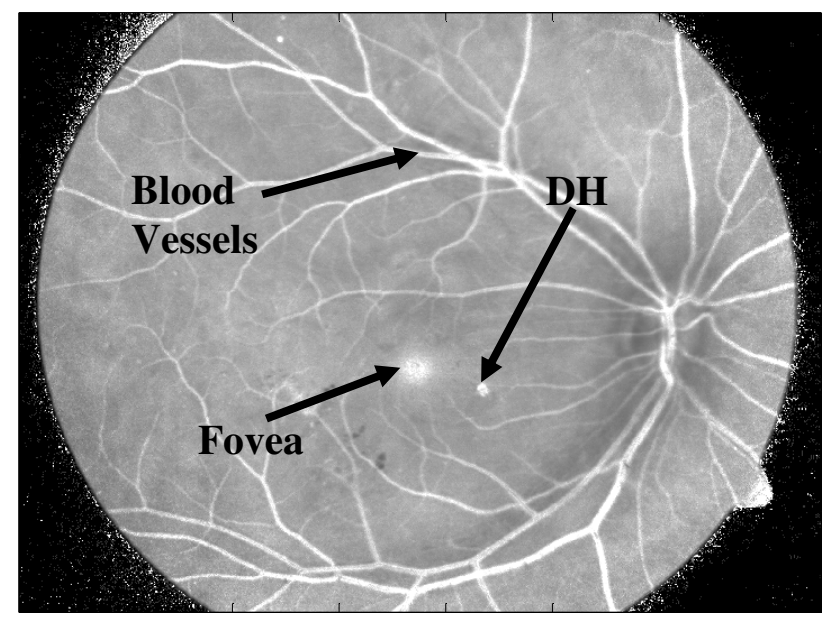

Figure 2: R/G intensities and identified $\mathrm{DH}$, fovea and blood vessels, which are clearly shown in this transformed image.

As with the exudate identification algorithm, the overall $\mathrm{DH}$ identification algorithm uses standard computer vision techniques in a new configuration, along with clinical observation or knowledge.

The overall algorithm is defined:

\section{Create Binary Image:}

- Calculate $\mathrm{R} / \mathrm{G}$ intensities from the fundus image

- Apply 50 pixel median filter scaling to $\mathrm{R} / \mathrm{G}$ intensities.

- Threshold top 7\% R/G intensities to yield binary image.

\section{Select Potential DHs by Shape:}

- Number all connected regions in binary image.

- For each connected region calculate the shape factor $\mathrm{F}_{\mathrm{s}}=$ $\mathrm{A} / \mathrm{L}_{\max }$, where $\mathrm{A}=$ area in pixels and $\mathrm{L}_{\max }=$ maximum distance between any 2 pixels in the region.

- Label all regions with 30 or more pixels and a shape factor greater than 0.54 as potential DH's.

\section{Check if Potential DHs are Blood Vessels:}

- For each potential DH find the line of minimum distance to the largest neighbouring connected region.

- Calculate the difference in intensity between the maximum and minimum intensities along the line of minimum distance.

- If difference between maximum and minimum intensities $<0.5$, the potential DH is unlabeled and connected to neighbouring region.

\section{Remove False DHs:}

- In a 40-pixel neighbourhood of each remaining $\mathrm{DH}$, compute the $\mathrm{R} / \mathrm{G}$ contours in steps of $0.05 \pm 0.5$ around the mean $\mathrm{R} / \mathrm{G}$ for the $\mathrm{DH}$.

- Let $r_{D H}$ denote the radius of the unique circle $C_{\text {circle }}$ precisely surrounding the $\mathrm{DH}$. Select a contour if:

a) Any part of contour is within $1.5 * r_{D H}$ from the centre of the circle

b) The contour has a length $>20$ pixels

- For each selected contour, calculate the mean absolute gradient of the surrounding points and choose the contour $\mathrm{C}$ corresponding to the largest value.

- Compute the distance $D_{c}$ between the current contour and nearest $\mathrm{DH}$. If $\mathrm{D}_{\mathrm{c}}>4$ pixels, reject the $\mathrm{DH}$.

\section{Output Number and Size of All DHs:}

\subsection{Analysis and Testing:}

The algorithms were developed base on fundus images from the DiaretdbO_v_1_1 database (Kauppi et al, 2006, 2007). Comparison is made to manual counting and identification of both DHs and exudates in each image. Results are measured in terms of: Specificity, Sensitivity, Positive Predictive Value (PPV) and Negative Predictive Value (NPV). All threshold values, such as filtered intensities, the number of pixels to form connected intensities, and the shape factor from the algorithm were determined from a selection of 6 images, three which were difficult images to diagnose and three that were straightforward. Accounting for the worst case scenarios that are selected by eye, avoids having to train the algorithms on excessive numbers of images. 
Diagnoses for these tests are based on existence, which only captures whether (or not) of these phenomena are present. Thus, R0 and M0 diagnoses indicate the existence of one or more DHs (R0) or exudates (M0). The absence of DHs and exudates are diagnosed $\mathrm{R} 1$ and $\mathrm{M} 1$, respectively. Thus, if the images are considered as being sequential over time, they would also diagnose the occurrence (or not) of new exudates and/or DHs, highlighting changes in the patient's DR status.

\section{RESULTS AND DISCUSSION}

\subsection{Hard Exudate Identification:}

The algorithm detected the presence of exudates correctly according to the diagnosis of (Kauppi et al, 2006, 2007) in all but two cases. The results are summarized in Table 1, with a sensitivity of $94.9 \%$ and specificity of $96.7 \%$. Note that to the human eye, the two false positives appeared to be exudates and the fact that dot haemorrhages are also present confirms this possibility. However, the images were pronounced clear by the diagnosis in (Kauppi et al, 2006, 2007). Similar results/controversy occurred for the two false negatives, demonstrating the inherent subjectivity involved in DR screening. Thus, no computer algorithm should be expected to fully agree with every ophthalmologist's observation in these types of cases, but do provide a consistent measure without subjectivity.

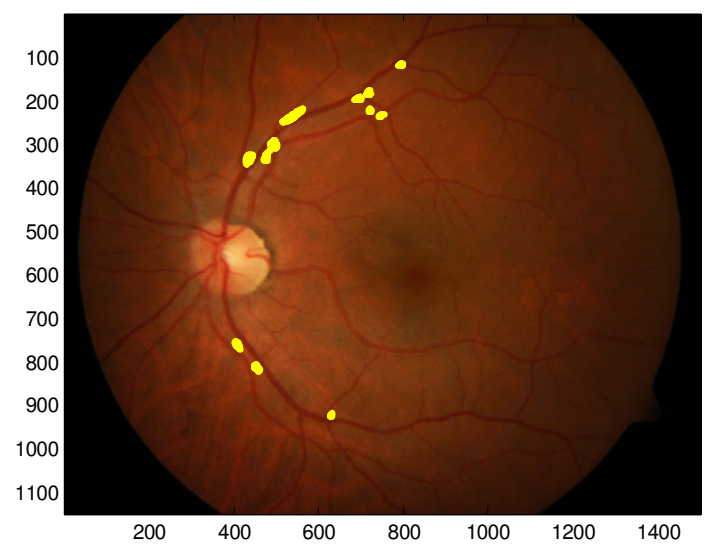

Figure 3: Image 099 (M1) showing false positives found when algorithm is used without Step IV.

Table 1: Hard exudate identification results

\begin{tabular}{|l|c|c|c|}
\cline { 2 - 3 } \multicolumn{1}{c|}{} & \multicolumn{2}{c}{ Observed Diagnosis } & \multicolumn{1}{c}{} \\
\cline { 2 - 3 } M1 & M1 & M0 & \\
\hline M0 & 59 & 2 & PPV 0.97 \\
\hline \multirow{2}{*}{} & 2 & 37 & NPV 0.95 \\
\hline $\begin{array}{c}\text { Specificity } \\
96.7 \%\end{array}$ & $\begin{array}{c}\text { Sensitivity } \\
94.9 \%\end{array}$ & \multicolumn{2}{c}{} \\
\cline { 2 - 3 } & &
\end{tabular}

One of the reasons for the significant accuracy of the method is the use of the contour method to remove false exudates. The contour method is based a little on the concept of level set methods to find closed boundaries in an image. A level set method evolves level 0 contours by solving a partial differential equation (PDE) until the object contour settles around the object or shape of interest. The stopping criteria is usually a region of high gradient. The contour method assumes that the image intensity contour surface already contains the required boundary around the shape of interest. This approach thus avoids having to solve a PDE which is very computationally expensive and not suitable for this application. The solution to the PDE is effectively the image intensity surface. To extract the final contour, the contour with essentially the largest gradient is chosen as detailed in the algorithms of the methodology. To the authors' knowledge this is a new approach in image processing which shows particular promise for DR.

Figure 3 shows the value of the contour checker in Step IV of the exudate algorithm, where an image (\#099) is diagnosed M1 or clear of exudates. The figure shows several identified regions along blood vessels where image contrast would have yielded false positive results without this added algorithm step. Note that simply eliminating all exudates within a tolerance of a blood vessel is not an effective solution, as exudates can lie close to veins and would be falsely removed. However, with the contour method, if an exudate is close to a vein the contour surrounds the exudate and thus does not remove it. Alternatively, around a false exudate the contour method finds the boundary of the vein, and pixels along this located boundary lie far away from the false exudate, leading to its removal in Step IV.

To demonstrate the overall efficacy of the algorithm, Figures 4 shows two images.
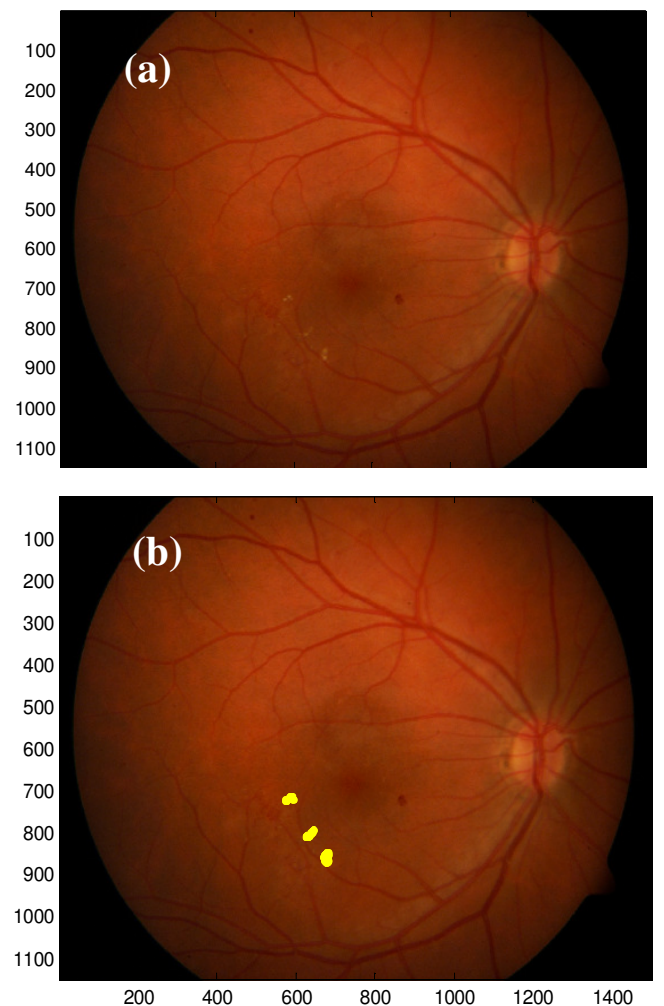

Figure 4: Specific before-after images of exudate identification algorithm results. 
The first is a standard fundus image and the second shows the identified hard exudates pixellated over top. It is clear that while they are easily found by eye with experience, they are not readily found at first glance. These marks match the results and diagnosis in the database.

More specifically, Figure 4 shows the original image in Figure $4 \mathrm{a}$ without processing and the identified image automatically generated from the algorithm. The 3 exudates are circled and pixellated in yellow in Figure $4 \mathrm{~b}$ after processing. Clinically, these three exudates are of significant size and would likely be considered as readily identifiable to a skilled or experienced technician.

Finally, speed and computational time are important. Each image in the 100 image database was processed with this algorithm in approximately 40-80 seconds. Each image had 2 Mpixels of data, where current fundus images can be much larger if required. However, the algorithm was also not optimised for speed. Thus a 1 minute, on average, processing time occurs. With optimisation or the use of $\mathrm{C}$ instead of Matlab, this time would be on the order of 1-10 seconds. Clinically, such turnaround times would enable the images to be taken and answer provided to the clinician, and expressed to the patient, in real-time while still in the office.

\subsection{DH Identification:}

For the first 100 from the DIARETDB0 database (Kauppi et al, 2006, 2007), the DH algorithm detected the presence of DHs accurately with no false positives and one false negative, as seen in Table 2. Again, the baseline for diagnosis was determined from the database and associated works.

Table 2: DH identification results.

\begin{tabular}{|c|c|c|c|}
\hline & \multicolumn{2}{|c|}{ Observed Diagnosis } & \\
\hline & R1 & R0 & \\
\hline R1 & 1 & 24 & PPV $96.0 \%$ \\
\hline R0 & 75 & 0 & NPV $100 \%$ \\
\hline & $\begin{array}{c}\text { Sensitivity } \\
98.7 \%\end{array}$ & $\begin{array}{c}\text { Specificity } \\
100 \%\end{array}$ & \\
\hline
\end{tabular}

The algorithm failed to correctly diagnose 1 image, which contained a DH as seen in Figure 5. However, the DH is seen to lie in a dark region of this particular image, as shown by the labelled section "C". As the DH lies in this dark region, the boundaries are not well defined. Therefore, when the fundus photograph is converted into a binary image, the shape of the DH becomes distorted and the shape factor, $F_{s}$, equation in Step II of the DH algorithm rules this DH out.

Without the contour checker, the algorithm found 5 false positives instead of 1 . But in a similar way to the case of the exudates, the contour method easily removed these false points. On the other hand, the contour method presently only works on regions that have been identified. Thus, it does not eliminate the false negative. However, simulations have shown that the contour method does not exclude these regions that were missed by the algorithm. Thus, future work should include the contour method in the initial identification part of the algorithm.

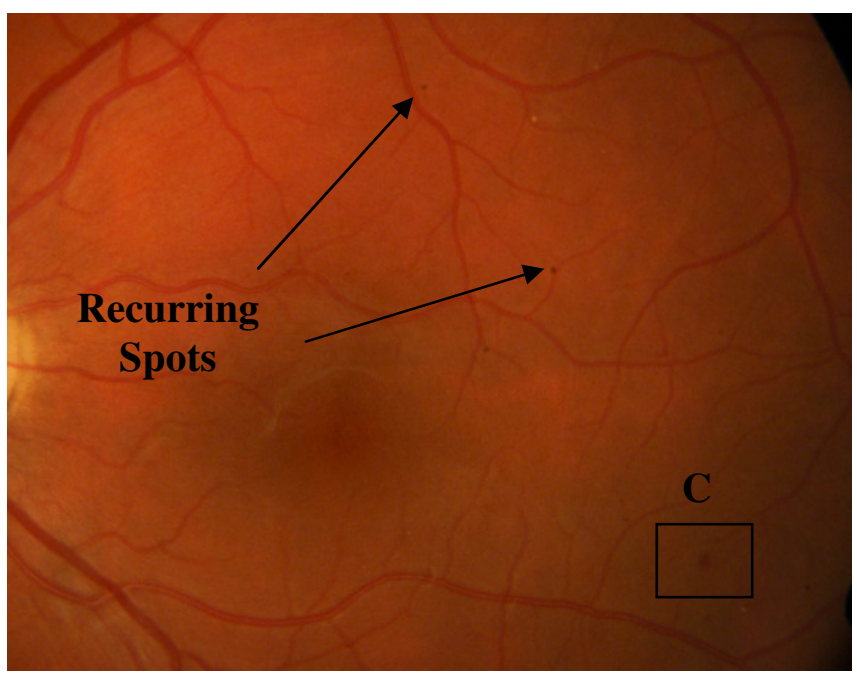

Figure 5: Close view of false negative ( $\mathrm{C}$ is missed, but found in the database) with recurring almost perfectly round spots not identified here or in (Kauppi et al, 2006, 2007).

Note that although there appears to be more than one $\mathrm{DH}$ in Figure 5, the recurring dark spots are not DH. These dark dots appear in almost all of the images in the database at the exact same location and are assumed to be associated with dust on the camera lens or similar imperfection. Other databases do not have such spots on their images, but also did not have diagnoses associated with their images. Hence, this image database was still used. Overall, these spots are ignored when processing the results of the algorithm. In practice, the quality of the lens could be checked, for example, by taking a picture of a white page.

\section{CONCLUSIONS}

Automatic methods for screening exudates and dot haemorrhages have been developed based on image processing methods that utilize colour, morphology and intensity gradients in fundus photographs. 100 images from a standard database were used to test the methods. For exudate detection, the sensitivity was $96.9 \%$ and specificity was $94.9 \%$ in terms of whether detecting the presence of DR or not. For dot haemorrhages, the sensitivity was $98.7 \%$ due to one false negative and the specificity was $100 \%$. From visual checks of the images, there were virtually no false exudates or dot haemorrhages detected which suggests the method could be used to accurately track changes over time. However, these results need to be validated in future clinical trials.

The reasons for the very high sensitivity with no false positives are the use of the red/ green ratio combined with a contour finding method and the image gradient after median filtering. The red/ green ratio has the advantages of helping to 
normalize out changes in light across the images and improves the contrast of DR features relative to the background. Furthermore, no statistical classifying methods were required.

The methods demonstrate a significant improvement over other algorithms in the literature and show potential for practical, clinical DR screening. The use of colour channels to directly identify DR dysfunctions allows clinical expertise and observation to be directly incorporated into the algorithm, providing a potentially far superior result.

Finally, it is also obvious that further clinical testing and trials will be required to prove the algorithm in practice. However, the same approach can be taken to a wider range of dysfunction or disease in the eye. Additionally, the ability to detect the number and size implies further clinical outcomes will be available in terms of tracking or monitoring patient evolution.

\section{REFERNCES}

Abramoff, MD Niemeijer, M Suttorp-Schulten, MSA Viergever, MA Russell, SR and Van Ginneken, B (2008). "Evaluation of a System for Automatic Detection of Diabetic Retinopathy From Color Fundus Photographs in a Large Population of Patients With Diabetes," Diabetes Care, 31(2):193-198.

Chia D, Yap E (2004). "Comparison of the Effectiveness of Detecting Diabetic Eye Disease: Diabetic Retinal Photography Versus Ophthalmic Consultation," Singapore Medical Journal, 45:276-279.

Ege BM, Hejlesen, OK, Larsen, OV, Moller, K, Jennings, B, Kerr, D and Cavan, DA (2000). "Screening for diabetic retinopathy using computer based image analysis and statistical classification," Computer methods and programs in Biomedicine, 62; pp. 165-175

ETDRSRG (Early Treatment Diabetic Retinopathy Study Research Group) (1991). "Early Photocoagulation for Diabetic Retinopathy," Ophthalmology, 98:766-785.

Fong D, Aiello L, Gardner T, King G, Blankenship G, Cavallerano J, Ferris F, Klein R (2003). "Diabetic Retinopathy," Diabetes Care, 26:226-229.

Fong D, Aiello L, Gardner T, King G, Blankenship G, Cavallerano J, Ferris F, Klein R (2004). "Retinopathy in Diabetes," Diabetes Care, 27:584-587

Frame, A.J., Undill, P.E., Cree,M.J., Olson, J.A.,McHardy, K.C., Sharp, P.F., Forrester, J.F. (1998). “A comparison of computer based classification methods applied to the detection of microaneurysms in opthalmic fluorescein angiograms," Computers in Biomedical Research, 28:225-238.

Gardner, GG, Keating, D, Williamson, TH and Elliott, AT (1996). "Automatic detection of diabetic retinopathy using an artificial neural network: a screening tool, ” $\mathrm{Br} J$ Ophthalmol, 80(11):940-944.

Hipwell J, Strachant F, Olson J, McHardy K, Sharp P, Forrester J (2000). "Automated detection of microaneurysms in digital red free photographs: a diabetic retinopathy screening tool," Diabetic Medicine, 17:588594.

Icks A, Trautner C, Haastert B, Breger M. Gianni G. (1997). "Blindness Due to Diabetes: Population based Age- and Sex- specific Incidence Rates," Diabetic Medicine, 14:571-575.

Kauppi, T, Kalesnykiene, V, Kamarainen, JK, Lensu, L, Sorri I, Uusitalo, H, Kalviainen, H, Pietila, J (2006). "Evaluation Database and Methodology for Diabetic Retinopathy algorithms," Technical Report, URL: http://www.it.lut.fi/ project/imageret/diaretdb0/.

Kauppi, T, Kalesnykiene, V, Kamarainen, JK, Lensu, L, Sorri I, Raininen, A, Voutilainen, R, Uusitalo, H, Kalviainen, H Pietila, J (2007). "The DIARETDB1 diabetic retinopathy database and evaluation protocol," Proc. of the British Machine Vision Conf (BMVC2007), Vol. 1, pp. 252-261.

Kinyoun, JL Martin, DC Fujimoto, WY and Leonetti, DL (1992). "Ophthalmoscopy Versus Fundus Photographs for Detecting and Grading Diabetic Retinopathy," Investigative Ophtalmology \& Visual Science, 33(6): 1888-1893.

Moss, SE, Klein, R, Kessler, SD, and Richie, KA (1985). "Comparison between ophthalmoscopy and fundus photography in determining severity of diabetic retinopathy," Ophthalmology, 92:62-67.

Sanchez, C, Hornero, R, Lopez, M, Aboy, M, Poza, J, Abasolo, P (2007). "A Novel Automatic Image Processing Algorithm for Detection of Hard Exudates based on Retinal Image Analysis," Medical Engineering and Physics, 30:350-357.

Sinthnayothin, C, Boyce, J, Williamson, T, Cook, H, Mensah, E, Lal, S, Usher, D. (2002). "Automated Detection of Diabetic Retinopathy on Digital Fundus Images," Diabetic Medicine, 19:105-112

TEDPRG (The Eye Disease Prevalence Research Group), (2004). "The Prevalence of Diabetic Retinopathy Among adults in the United States," Archives of Ophthalmology, 122:552-563.

Walter, T, Massin, P, Erginay, A, Ordonez, R, Jeulin, C, Klein, J (2007). "Automatic Detection of Microaneurysms in Colour Fundus Images," Medical Image Analysis, 11:555-566.

Wild, S, Roglic, G, Green, A, Sicree, R, King, H (2004). "Global Prevalence of Diabetes: Estimates for the year 2000 and projections for 2030," Diabetes Care, 27:10471053 